\title{
Incidence of chronic pain and its relation to quality of life three months after ICU discharge
}

\author{
M. CHANZA 1 , A. Ojeda1, D. Sanchez ${ }^{1}$, A. Calvo1, V. Zafra'², S. Belmonte ${ }^{3}$ \\ ${ }^{1}$ Department of Anesthesiology. Hospital Clinic de Barcelona - Barcelona (Spain), ${ }^{2}$ Department of Nursing. Hospital Clinic de \\ Barcelona - Barcelona (Spain), ${ }^{3}$ Phisiotherapist. Department of Medicine. Hospital Clinic de Barcelona - Barcelona (Spain)
}

\section{BACKGROUND}

Previous studies have described an increase in chronic pain in patients who have been admitted to the ICU. Furthermore, many patients present a deterioration in their quality of life after ICU discharge, increasing health resources consumption. These patients are susceptible to developing a Post-ICU Syndrome (PICS).

\section{OBJECTIVES}

The aim of our study is to determine the incidence of chronic pain 3 and 12 months after ICU discharge. The second aim is to determine the impact on quality of life by analyzing anxiety, depression, cognitive impairment and post-traumatic stress disorder.

\section{METHODS}

A prospective observational study was started in March, 2017. Patients with written informed consent were evaluated three times: at ICU discharge, at 3 and 12 months after ICU discharge. Exclusion criteria included neurological and psychiatric pathology, and history of substance abuse. We enrolled medical and surgical patients discharged after a minimun of 2 days in the ICU.

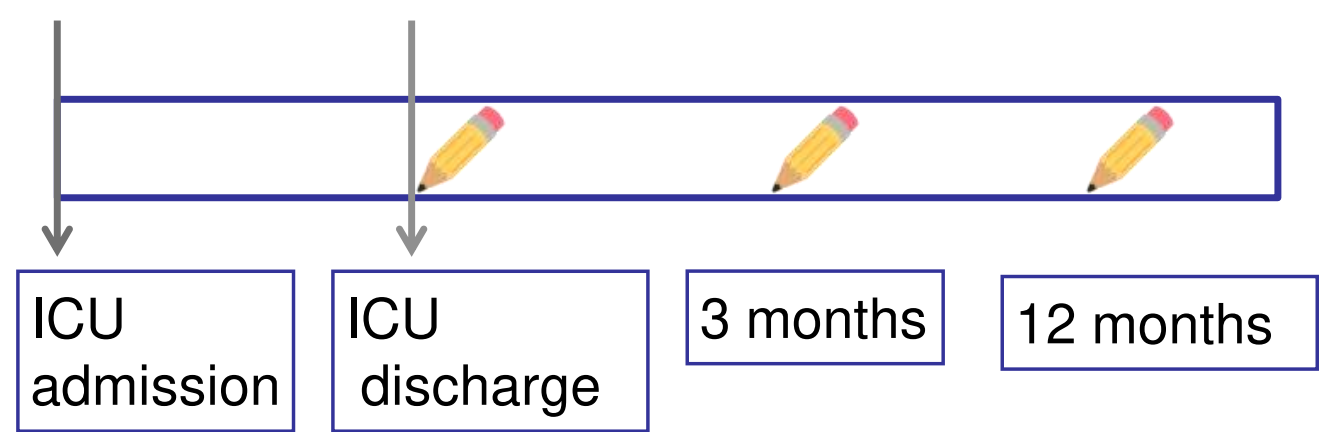

Numeric Rating Scale
(NRS) for pain
Neuropathic pain (DN4
Scale)
Anxiety and Depression
(HAD test)
Quality of life
(EuroQol-5D test )

Table 1. Data collected in questionnaire
Social support (Duke test )

Cognitive impairment (Pfeiffer test )

Brief Pain interference scale (BPI scale)

Post-traumatic Stress Disorder (PTSD) test

Figure 1.Time line

\section{RESULTS}

Preliminary results until March 2018 are presented

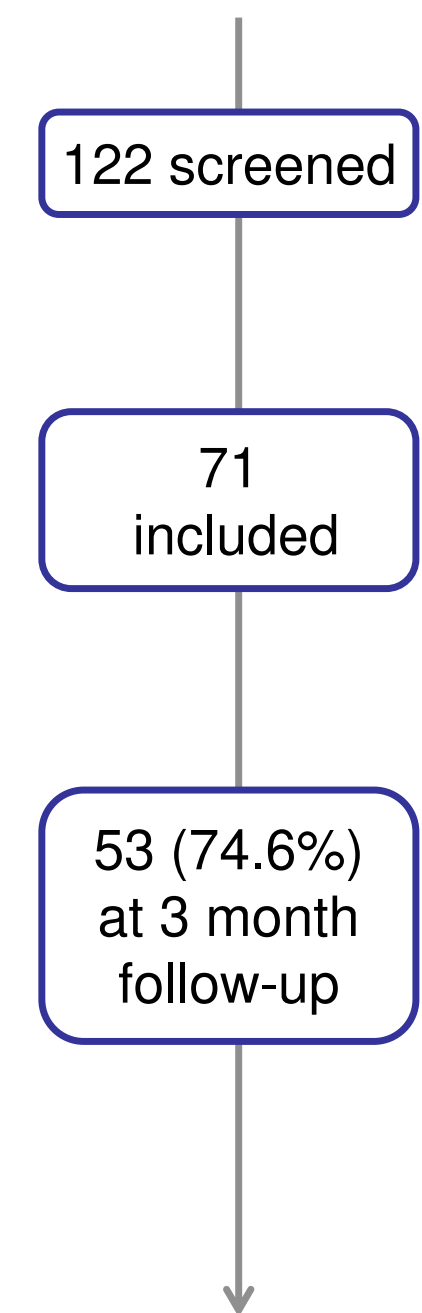

Figure 2. Flowchart patient recruiment.

\begin{tabular}{|cc|}
\hline Variable & Median $\left({ }_{\text {Q1-Q3 }}\right)$ \\
\hline Age & $63(19-87)$ \\
\hline
\end{tabular}

APACHE II score

$14(3-32)$

Charlson

comorbidity

Index

ICU stay

$5(3-38)$

Sepsis $36 \%$

$77 \%$

Mechanical

$37 \%$

ventilation

3 month mortality

$18 \%$

Table 2. Demographic data

$4(0-15)$

(1)

\section{CONCLUSIONS}

This is the first study that evaluates pain level at ICU discharge and its subsequent evolution. Results suggest an increase in the incidence of chronic pain and also of neuropathic pain with differences statistically significant. Anxiety, depression and PTSD are presented in these patients. A higher interference in the activities of daily life owing to higher pain level has been identified in patients. Patients also have a perception of worse health. Therefore, the inclusion of chronic pain in the definition of Post Intensive Care Syndrome should be considered.

\section{References}

1. Kyranou M, Puntillo K. The transition from acute to chronic pain: might intensive care unit patients be at risk? Annals of Intensive Care 2012,2:36

2. Jackson JC, Pandharipande PP, Girard TD, Brummel NE, Thompson JL Hughes CG et al. Depression, Post-traumatic Stress Disorder, and Functional Disability in Survivors of Critical Illness: results from the BRAIN ICU (Bringing to light the Risk Factors And Incidence of Neuropsychological dysfunction in ICU survivors) Investigation: A Longitudinal Cohort Study. Lancet Respir Med. 2014 May; 2(5): 369-37 